\title{
MD simulations of total X-ray scattering in protein crystals
}

\author{
David A. Case, ${ }^{a}$ Stephen P. Meisburger ${ }^{b}$ and Nozomi Ando ${ }^{b}$ \\ ${ }^{a}$ Dept. of Chemistry and Chemical Biology, Rutgers University, Piscataway, NJ 08854 USA, david.case@rutgers.edu \\ ${ }^{b}$ Dept. of Chemistry and Chemical Biology, Cornell University, Ithaca, NY 14853 USA
}

The adaption of graphical processing units (GPUs) to biomolecular simulations has made microsecondscale simulations of biomolecular crystals available on a nearly-routine basis [1-5]. Typically, a super-cell consisting of several crystallographic unit cells becomes the periodically-repeating unit in the simulation; in this talk, I will consider super-cells with up to 343 unit cells. We have collected synchrotron data and have carried out multi-microsecond simulations of crystals of lysozyme (in three space groups), insulin, thaumatin, apoferritin and myoglobin. Here are some ways this data might be used:

- Straightforward comparisons between computed and experimental average structures and atomic displacement parameters can be used to identify problems in biomolecular force fields [1-4]. The high accuracy and precision crystallography (compared to NMR or other solution methods) makes such comparisons uniquely informative, and the statistics of such comparisons benefit from having many copies of chains in equivalent environments in the simulation.

- Structural fluctuations in the simulations can be used to estimate diffuse scattering intensities, which can be compared to measurements using modern detectors $[5,6]$. Both simulations and experiments allow one to place Bragg and non-Bragg intensities on a common, "absolute", scale, showing that a surpisingly large fraction of scattered X-rays occur between the Bragg peaks, and are usually ignored.

- Simulations provide a model for density fluctuations in regions of "disordered" or "bulk" solvent (mainly water). Such models appear to account for solvent contributions to Bragg intensities in ways that are a systematic improvement over the procedures used in most protein structure refinement protocols.

- Simulations provide a plausible, if imperfect, model for conformational heterogeneity in biomolecular crystals. We find that thermally-activated phonon-like modes, "trapped" lattice distortions, and more localized protein and solvent conformational disorder all contribute to diffuse scatter. We are still exploring ways to make the best comparisons with experimental data.

[1] D.S. Cerutti, P.L. Freddolino, R.E. Duke and David A. Case. Simulations of a protein crystal with a high resolution X-ray structure: Evaluation of force fields and water models. J. Phys. Chem. B 114, 12811-12824 (2010).

[2] P.A. Janowski, D.S. Cerutti, J. Holton and D.A. Case. Peptide crystal simulations reveal hidden dynamics. J. Am. Chem. Soc. 135, 7938-7948 (2013).

[3] P.A. Janowski, C. Liu and D.A. Case. Molecular dynamics of triclinic lysozyme in a crystal lattice. Prot. Sci. 25, 87-102 (2016).

[4] D.S. Cerutti and D.A. Case. Molecular dynamics simulations of macromolecular crystals. Wires Comput. Mol. Sci. 8, e1402 (2018).

[5] A.H. Van Benschoten, L. Liu, A. Gonzalez, A.S. Brewster, N.K. Sauter, J.S. Fraser and M.E. Wall. Measuring and modeling diffuse scattering in protein X-ray crystallography. Proc. Natl. Acad. Sci. USA 113, 4069-4074 (2016); M.E. Wall. Internal protein motions in molecular-dynamics simulations of Bragg and diffuse X-ray scattering. IUCrJ 5, 172-181 (2018).

[6] S.P. Meisburger and N. Ando. Correlated Motions from Crystallography beyond Diffraction. Acc. Chem. Res. 50, 580-583 (2017). 